\title{
CORRECTION APPLIED TO THE CARRIER FREQUENCIES OF THE RADIO \\ STATIONS GBR AND RES ACCORDING TO THE USSR STATE \\ STANDARD OF TIME AND FREQUENCY
}

TABLE 1. Corrections Applied to the Carrier Frequencies of the Radio Stations GBR and RES According to the USSR State Standard of Time and Frequency

\begin{tabular}{|c|c|c|c|c|c|c|c|c|}
\hline \multicolumn{9}{|c|}{ April, 1970} \\
\hline Date & $\begin{array}{l}\mathrm{GBR}, 16 \mathrm{kHz} \\
\text { referred to } \\
0.3^{\mathrm{h}} \mathrm{TU}\end{array}$ & $\begin{array}{l}\mathrm{RES}, 100 \\
\mathrm{kHz} \text { referred } \\
\text { to } 19^{\mathrm{h}} \mathrm{TU}\end{array}$ & Date & $\begin{array}{l}\mathrm{GBR}, 16 \mathrm{kHz}^{\mathrm{H} z} \\
\text { referred to } \\
0.3^{\mathrm{h}} \mathrm{TU}\end{array}$ & $\begin{array}{l}\mathrm{RES}, 100 \\
\mathrm{kHz} \text { referred } \\
\text { to } 19^{\mathrm{h}} \mathrm{TU}\end{array}$ & Date & $\begin{array}{l}\mathrm{GBR}_{2} 16 \mathrm{kHz} \\
\text { referred to } \\
0.3^{\mathrm{h}} \mathrm{TU}\end{array}$ & $\begin{array}{l}\text { RES, } 100 \\
\text { WHz referred } \\
\text { to } 19^{h} \mathrm{TU}\end{array}$ \\
\hline $\begin{array}{r}1 \\
2 \\
3 \\
4 \\
5 \\
6 \\
7 \\
8 \\
9 \\
10\end{array}$ & $\begin{array}{l}-300,4 \cdot 10^{-10} \\
-300,5 \\
-300,4 \\
-300,6 \\
-300,1 \\
-300,5 \\
-300,8 \\
-300,4 \\
-300,9 \\
-300,6\end{array}$ & $\begin{array}{l}-300,1 \cdot 10^{-10} \\
-300,1 \\
-299,9 \\
-300,0 \\
-300,3 \\
-300,3 \\
-300,2 \\
-300,2 \\
-300,0 \\
-300,2\end{array}$ & $\begin{array}{l}11 \\
12 \\
13 \\
14 \\
15 \\
16 \\
17 \\
18 \\
19 \\
20\end{array}$ & $\begin{array}{l}-300,5 \cdot 10^{-10} \\
-300,5 \\
-300,3 \\
-300,7 \\
-300,5 \\
-300,0 \\
-300,3 \\
-300,4 \\
-300,4 \\
-300,8\end{array}$ & $\begin{array}{l}-300,3 \cdot 10^{-10} \\
-300,2 \\
-300,0 \\
-300,0 \\
-300,2 \\
-300,1 \\
-299,8 \\
-300,0 \\
-300,1 \\
-300,1\end{array}$ & $\begin{array}{l}21 \\
22 \\
23 \\
24 \\
25 \\
26 \\
27 \\
28 \\
29 \\
30\end{array}$ & $\begin{array}{l}-300,6 \cdot 10^{-10} \\
-300,5 \\
-300,0 \\
-300,4 \\
-300,6 \\
-300,5 \\
-300,7 \\
-300,5 \\
-300,6 \\
-300,5\end{array}$ & $\begin{array}{l}-300,1 \cdot 10^{-10} \\
-300,2 \\
-299,2 \\
-297,9 \\
-300,9 \\
-301,2 \\
-301,6 \\
-300,6 \\
-300,3 \\
-300,1\end{array}$ \\
\hline & & & & & Mean & . . & $-300,5$ & $-300,2$ \\
\hline
\end{tabular}

Translated from Izmeritel 'naya Tekhnika, No. 7, p. 85, July, 1970.

(C) 1970 Consultants Bureau, a division of Plenum Publishing Corporation, 227 West 17th Street, New York, N.Y. 10011. All rights reserved. This article cannot be reproduced for any purpose whatsoever without permission of the publisher. A copy of this article is available from the publisher for $\$ 15.00$. 